\title{
Patient-oriented research: a new model for anesthesia in the 2lst century?
}

\author{
Oliver Boney, MA (Cantab), MBBS (Hons), FRCA $₫$
}

Received: 10 February 2020/Revised: 10 February 2020/Accepted: 10 February 2020/Published online: 10 March 2020

(C) Canadian Anesthesiologists' Society 2020

No matter how complicated the research, or how brilliant the researcher, patients and the public always offer unique, invaluable insights. Their advice when designing, implementing and evaluating research invariably makes studies more effective, more credible and often more cost efficient as well.

Dame Sally Davies, UK Chief Medical Officer 2010-2019

In recent years, the notion that medical research should involve patients as active participants at every stage has gained increasing traction. Growing evidence of a mismatch between patients and researchers regarding which research topics they consider most important, and increasing recognition that the medical model of research may not always produce research that maximizes benefits to patients, ${ }^{1,2}$ have seen the "patient-oriented" research movement emerge as a new paradigm for developing research strategies.

Furthermore, the widespread endorsement of the patientoriented research model across the Western world is reflected in the various new institutions created to support and promote it. The Patient-Centred Outcomes Research Institute (PCORI) in the US (https://www.pcori.org/), the United Kingdom's (UK) NIHR INVOLVE (https://www. invo.org.uk/), and the Canadian Institutes of Health Research Strategy for Patient Oriented Research (SPOR) units (https://cihr-irsc.gc.ca/e/41204.html) have all emerged in the past decade. These are all backed by

O. Boney, MA (Cantab), MBBS (Hons), FRCA ( $\square$ )

The Royal London Hospital, Whitechapel, London, UK

e-mail: oliverboney@hotmail.com

National Institute of Academic Anaesthesia Health Services Research Centre, Churchill House, 35 Red Lion Square, London, England WC1R 4SG, UK significant public funding, with the explicit aim of fostering more patient and public participation in all aspects of medical research.

Anesthesia has, however, been slower than other specialties to embrace patient collaboration in research design and development, despite increasing recognition that anesthesia-and certainly perioperative medicinecannot meaningfully focus solely on patient care delivered within the operating room. ${ }^{3}$ Nevertheless, our specialty is also belatedly recognizing the value of increasing patient participation in shaping a truly inclusive research agenda that reflects the priorities of all stakeholders. ${ }^{4}$ The Canadian Anesthesia Research Priority Setting Partnership (CAR PSP), reported in this issue of the Journal, ${ }^{5}$ is a timely illustration of the anesthesia research community's acceptance of patient-oriented research. Or is it?

McKeen et al. report the results of a collaborative "research prioritisation" process to which patients, carers, researchers, and clinicians from across Canada were invited to contribute. ${ }^{5}$ They followed the methodology proscribed by the James Lind Alliance (JLA), a UK-based non-profit organisation funded by the UK National Institute for Health Research (NIHR), whose methods have been used in over a hundred research "Priority Setting Partnerships" (PSPs) in many different areas of healthcare, ${ }^{6}$ including the UK's own Anesthesia and Perioperative Care PSP undertaken in 2014-2015. ${ }^{7}$

While I commend the authors on the ambitious scope of the CAR PSP-namely to "suggest priorities for the Canadian anesthesia research agenda"-several issues warrant further consideration. To begin with, how valid is the JLA's methodology to the CAR PSP? Is the process translatable from the UK to Canada without any modifications? For example, the authors describe several 
methodological challenges, most of which were also encountered in the earlier UK exercise. These included difficulties engaging "hard-to-reach" minority groups, efforts to maximize the balance of different stakeholder viewpoints, and the broad scope of a PSP. Indeed, the CAR PSP did not focus on a specific disease area with a defined patient population but instead reached out to all members of the public who have ever had a procedure requiring anesthetic intervention, their caregivers, and all healthcare professionals (not just anesthesiologists) involved in a patient's perioperative journey.

Inevitably, a collaborative venture of this nature can never be described as fully representative of all its constituent stakeholders. Nevertheless, most authorities recognize that a PSP probably achieves adequate representation as long as some contributions are received from all the main stakeholder groups, and as long as all contributions are given equal weight. ${ }^{8}$ Similarly, while achieving an even balance of different stakeholder views may be challenging, measures to ensure a fair and transparent consensus process, with roughly even numbers of participants from different stakeholder groups, helps to minimize any biases. The CAR PSP clearly adopted appropriate measures to engage minority groups and to ensure a balance of stakeholder viewpoints; in a country of the size and diversity of Canada, their success on this front is all the more commendable.

Another commonly cited criticism of the JLA process, which perhaps applies especially to a PSP concerning such a broad area of healthcare, is that the top ten research questions produced are too general and non-specific. While this is largely an inevitable consequence of the JLA methodology—whereby all "raw" research suggestions received are synthesized into a shortlist of "summary" research questions-one can imagine that a research funding body wishing to fund high priority research might not know where to begin when faced with the CAR PSPs number one priority: to determine "Which factors before, during, and after receiving anesthesia for surgery are most important to improve patient outcomes and satisfaction?" This question is simply too broad to guide research funders in assessing competing grant applications, since almost any proposed clinical trial could be deemed to address this question.

Previous PSPs in other healthcare areas have reported similar uncertainty from funding bodies about how to focus a PSP's often rather unfocused research themes into research-ready hypotheses. ${ }^{9}$ Moreover, the refinement of raw research suggestions into a shortlist of "indicative" research questions is a subjective process that often involves rewording of questions, amalgamation of similar themes, the finer details of which have been shown to vary significantly between different PSPs. ${ }^{10}$ While the CAR PSP appears to have followed the JLA methodological principles in reducing a list of $500+$ suggestions into a manageable shortlist of 49 , this area of JLA methodology clearly requires further scrutiny. This additional scrutiny is needed both to reduce the subjectivity inherent in the process and to avoid producing questions that are overly general and non-specific in nature.

Meanwhile, the CAR PSP also highlights some important questions for the Canadian anesthesia research community going forward. Who "owns" these research priorities, and how are they to be used? How will the impact of the CAR PSP be measured? And perhaps most importantly, does the patient-oriented research model yield better or more productive anesthesia research than the current medical model of selecting research topics?

Following a recent evaluation of the past decade's PSP outputs, the JLA recommends that explicit strategies are agreed at the start of a PSP for "what to do" with the ensuing top research priorities-i.e., that the PSP's funders have a clear plan for precisely how their research priorities will be endorsed by professional and/or research organisations, submitted to funding bodies, and what responsibility those organisations will have (if any) for addressing them. ${ }^{11}$ Previous PSPs have, however, met with variable success in showing the impact of their outputs. ${ }^{12,13}$ Again, this is an area of ongoing development in JLA methodology that perhaps warrants more attention, particularly given the JLA's stated purpose of reducing waste in research and maximizing its value to patients. ${ }^{14}$

In summary, the CAR PSP authors are to be congratulated on the ambitious scope of this work, and for achieving a robust consensus about anesthesia research priorities that reflects views from a wide variety of stakeholders across Canada. They have clearly followed the JLA methodology, which is widely regarded as the current gold standard for collaborative research agenda setting. Nevertheless, the impact of the identified research priorities on the future anesthetic research agenda remains to be seen, and the value of research priority setting exercises in informing decisions about what anesthesia research gets funded-whether in Canada or the UK-is likewise uncertain. Regardless of their eventual impact on research funding decisions, it is hopeful that the CAR PSP (and the earlier UK version) will also spark further debate regarding the role of patient-oriented research in anesthesia and perioperative medicine. While few would argue that anesthesia research should not ultimately seek to improve outcomes for patients, patient and public time and energy is not an infinite resource. It is therefore incumbent on anesthesia researchers to collaborate with patients where their participation will genuinely improve the design, development, or dissemination of research. The CAR PSP has uniquely shown both the benefits and challenges of 
patient participation on a national scale. This important undertaking should therefore shape the evolution of both patient-oriented and overall anesthesia research agendas.

\section{La recherche axée sur le patient, un nouveau modèle pour l'anesthésie du $21^{\mathrm{e}}$ siècle?}

Peu importe la complexité de la recherche ou l'érudition du chercheur, les patients et le grand public introduisent toujours des perspectives à la fois uniques et inestimables. Leurs conseils pour la conception, la mise en œuvre et l'évaluation de la recherche rendent systématiquement les études plus efficaces, plus crédibles - et bien souvent plus rentables.

Dame Sally Davies, médecin en chef du RoyaumeUni, 2010-2019

Au cours des dernières années, l'idée que la recherche médicale devrait impliquer les patients en tant que participants actifs à tous les stades a gagné beaucoup de terrain. De plus en plus de données probantes mettent en lumière le décalage entre patients et chercheurs à propos des thèmes de recherche que les uns et les autres considèrent les plus importants; qui plus est, nous assistons à une prise de conscience croissante que le modèle médical de recherche pourrait ne pas toujours produire des recherches optimisant les bienfaits pour les patients. ${ }^{1,2}$ C'est pourquoi un mouvement de recherche «axée sur le patient» a vu le jour comme nouveau paradigme de mise au point de stratégies de recherche.

En outre, le soutien très prononcé pour le modèle de recherche axée sur le patient dans le monde occidental se reflète dans diverses nouvelles institutions créées afin de le soutenir et de le promouvoir. Le PCORI (Patient-Centred Outcomes Research Institute - Institut de recherche sur les devenirs axés sur le patient) aux États-Unis, le programme INVOLVE du NIHR au Royaume-Uni (https://www.invo. org.uk/) et les unités de la Stratégie de recherche axée sur le patient (SRAP) des Instituts de recherche en santé du Canada (https://cihr-irsc.gc.ca/f/41204.html) ont tous vu le jour au cours des dix dernières années. Ces initiatives sont toutes soutenues par un important financement public, et leur but avoué est de favoriser une plus grande participation des patients et du grand public dans tous les aspects de la recherche médicale.
L'anesthésie a toutefois été plus lente que d'autres spécialités à embrasser la collaboration du patient dans la conception et la mise en œuvre de la recherche, malgré la prise de conscience toujours plus grande que l'anesthésie et la médecine périopératoire en général - ne peut pas véritablement se concentrer exclusivement sur les soins aux patients prodigués en salle d'opération. ${ }^{3}$ Notre spécialité prend toutefois conscience, sur le tard, de la valeur ajoutée d'une plus importante participation du patient pour former un agenda de recherche véritablement inclusif qui reflète les priorités de tous les acteurs. ${ }^{4}$ Le Partenariat pour l'établissement des priorités pour la recherche en anesthésie au Canada (CAR PSP), dont il est question dans ce numéro du Journal, ${ }^{5}$ constitue un exemple à point nommé de l'acceptation de la communauté de recherche en anesthésie de la recherche axée sur le patient. Ou l'est-il vraiment?

McKeen et coll. rapportent les résultats d'un processus collaboratif de «priorisation de la recherche » auquel patients, soignants, chercheurs et cliniciens des quatre coins du Canada ont été invités à participer. ${ }^{5}$ Les auteurs ont suivi la méthodologie prescrite par l'Alliance James Lind (JLA), un organisme à but non lucratif basé au Royaume-Uni et financé par l'Institut national de recherche en santé britannique (NIHR), dont les méthodes ont été utilisées dans plus d'une centaine de «Partenariats pour l'établissement des priorités » (ou PSP, pour Priority Setting Partnership) en recherche dans plusieurs domaines différents des soins de santé, ${ }^{6}$ notamment pour le PSP en anesthésie et soins périopératoires britannique, entrepris en 2014-2015. ${ }^{7}$

Bien que je tienne à féliciter les auteurs pour l'objectif ambitieux du CAR PSP - soit d' " indiquer les priorités de l'agenda de recherche en anesthésie au Canada », plusieurs éléments méritent une réflexion plus approfondie. Tout d'abord, dans quelle mesure la méthodologie de la JLA estelle pertinente pour le CAR PSP? Le processus est-il transférable du Royaume-Uni au Canada sans y apporter de modifications? Par exemple, les auteurs décrivent plusieurs difficultés méthodologiques, dont la plupart avaient déjà été rencontrées dans la version britannique précédemment réalisée. Parmi ces difficultés, citons celle d'impliquer les groupes minoritaires isolés, "difficiles à rejoindre », les efforts visant à maximiser l'équilibre entre les points de vue des différents acteurs, et la portée très large d'un PSP. En effet, le CAR PSP ne s'est pas concentré sur un domaine pathologique spécifique avec une population de patients définie, mais a plutôt tenté de rejoindre tous les membres du grand public qui ont subi une intervention nécessitant une intervention anesthésique, leurs aidants, et tous les professionnels de la santé (pas seulement les anesthésiologistes) impliqués dans le parcours périopératoire d'un patient. 
Inévitablement, une entreprise collaborative de cette nature ne pourra jamais être décrite comme représentant pleinement tous les acteurs qui la constituent. Toutefois, la plupart des instances reconnaissent qu'un PSP atteint probablement une représentation adéquate tant qu'il reçoit des contributions de tous les groupes d'acteurs, et tant que toutes les contributions sont considérées de valeur égale. ${ }^{8}$ De la même manière, bien qu'il puisse être difficile d'atteindre un équilibre juste entre les points de vue des différents acteurs, les mesures visant à garantir un processus de consensus équitable et transparent, comptant un nombre plus ou moins égal de participants dans tous les groupes concernés, aident à minimiser les biais. Le CAR PSP a clairement adopté les mesures adaptées pour impliquer les groupes minoritaires et garantir un équilibre entre les divers points de vue; dans un pays de la taille et de la diversité du Canada, leur réussite à ce point de vue est d'autant plus louable.

Une autre critique fréquemment citée du processus de la JLA, qui s'applique peut-être particulièrement à un PSP touchant un champ tellement vaste des soins de santé, est que les dix questions de recherche prioritaires produites sont trop générales et manquent de spécificité. Alors qu'il s'agit là largement d'une conséquence inévitable de la méthodologie de la JLA - dans laquelle toutes les suggestions «brutes» de recherche sont résumées dans une présélection de questions de recherche «sommaires », - on peut imaginer qu' un organisme de financement pour la recherche souhaitant soutenir des recherches de priorité élevée pourrait ne pas savoir par où commencer lorsqu'il fait face à la priorité numéro un des CAR PSP, qui est de déterminer «Quels facteurs avant, pendant et après avoir reçu une anesthésie pour une chirurgie sont les plus importants pour améliorer les devenirs et la satisfaction des patients? »Cette question est évidemment trop générale pour orienter les organismes de financement de la recherche lors de l'évaluation de demandes de bourse concurrentes, étant donné que pratiquement toutes les études cliniques proposées pourraient être jugées comme traitant de cette question.

Les PSP précédents réalisés dans d'autres domaines des soins de santé ont également fait état d'une même hésitation des organismes de financement quand il était question de savoir comment concentrer les thèmes de recherche souvent vastes des PSP en des hypothèses prêtes pour la recherche. 9 Qui plus est, le peaufinage de suggestions de recherche brutes en une présélection de questions de recherche «indicatives » est un processus subjectif qui implique souvent de réécrire les questions, d'amalgamer des thèmes semblables, et il a été démontré que cela peut varier beaucoup d'un PSP à l'autre. ${ }^{10}$ Bien que le CAR PSP semble avoir respecté les principes méthodologiques de la JLA en réduisant une liste de plus de 500 suggestions en une présélection plus gérable de 49 questions, ce domaine de la méthodologie de la JLA nécessite clairement un approfondissement. Cet approfondissement est nécessaire tant pour réduire la subjectivité inhérente au processus que pour éviter de produire des questions de nature trop généraliste et non spécifique.

En attendant, le CAR PSP met également en lumière certaines questions importantes pour la communauté de recherche en anesthésie canadienne pour l'avenir. À qui « appartiennent » ces priorités de recherche, et comment doivent-elles être utilisées? Comment l'impact du CAR PSP sera-t-il mesuré? Et, question peut-être encore plus fondamentale, le modèle de recherche axée sur le patient donne-t-il une recherche en anesthésie de meilleure qualité ou plus productive que le modèle médical actuel de sélection des thèmes de recherche?

À la suite d'une évaluation récente des résultats des PSP des dix dernières années, la JLA recommande que des stratégies explicites soient convenues au début d'un PSP sur «quoi faire » avec les priorités de recherche subséquentes les plus importantes - en d'autres termes, il faut que les agences finançant les PSP disposent d'un plan clair décrivant la façon dont leurs priorités de recherche seront promues par les organisations professionnelles et/ou de recherche, la manière dont elles seront soumises aux organismes de financement, et la responsabilité que ces organisations auront (si elles en ont) d'y répondre. ${ }^{11}$ Les PSP précédents ont toutefois eu un succès variable dans la démonstration de l'impact de leurs résultats. ${ }^{12,13}$ Encore une fois, il s'agit là d'une zone d'amélioration continue dans la méthodologie de la JLA qui aurait peut-être besoin de davantage d'attention, particulièrement étant donné l'objectif déclaré de la JLA de réduire le gaspillage dans la recherche et de maximiser sa valeur pour les patients. ${ }^{14}$

En résumé, les auteurs du CAR PSP doivent être félicités pour la nature ambitieuse de leurs travaux, et pour avoir atteint un consensus solide quant aux priorités de recherche en anesthésie qui reflètent les points de vue d'une grande variété d'intervenants partout au Canada. Ils ont clairement suivi la méthodologie de la JLA, considérée de manière générale comme la référence en matière d'établissement de priorités de recherches collaboratives. Toutefois, l'impact des priorités de recherche identifiées sur le calendrier futur de recherche en anesthésie reste encore à déterminer, et la valeur des exercices d'établissement des priorités de recherche pour informer les décisions concernant les sujets qui obtiendront du financement pour la recherche en anesthésie - que ce soit au Canada ou au Royaume-Uni - est tout aussi incertaine. Indépendamment de leur impact final sur les décisions de financement de la recherche, nous espérons que le CAR PSP (et sa version britannique précédente) alimentera 
également un débat approfondi sur le rôle de la recherche axée sur le patient en anesthésie et en médecine périopératoire. Alors que la plupart d'entre nous sommes d'accord que l'un des buts ultimes de la recherche en anesthésie est d'améliorer les devenirs des patients, le temps et l'énergie des patients et du grand public ne sont pas infinis. Il incombe donc aux chercheurs en anesthésie de collaborer avec les patients dans les domaines où leur participation améliorera véritablement la conception, la mise au point ou la diffusion de la recherche. Le Partenariat pour l'établissement des priorités pour la recherche en anesthésie au Canada a su montrer tant les avantages que les défis liés à la participation de patients à l'échelle nationale. Cette entreprise d'envergure devrait donc façonner l'évolution des agendas de recherche axés sur le patient et en anesthésie.

\section{Conflicts of interest None.}

Disclosure Dr. Boney was the Steering Group Coordinator for the UK Anaesthesia and Perioperative Care PSP.

\section{Funding statement None.}

Editorial responsibility This submission was handled by Dr. Hilary P. Grocott, Editor-in-Chief, Canadian Journal of Anesthesia.

\section{Conflit d'intérêt Aucun.}

Déclaration Dr Boney était le coordonnateur du Groupe de pilotage pour le PSP en anesthésie et soins périopératoires du Royaume-Uni.

\section{Déclaration de financement Aucune.}

Editorial responsibility Cet article a été traité par Dr Hilary P. Grocott, rédacteur en chef, Journal canadien d'anesthésie.

\section{References}

1. Crowe S, Fenton M, Hall M, Cowan K, Chalmers I. Patients', clinicians' and the research communities' priorities for treatment research: there is an important mismatch. Res Involv Engagem 2015. DOI: https://doi.org/10.1186/s40900-015-0003-x.

2. Liberati $A$. Need to realign patient-oriented and commercial and academic research. Lancet 2011; 378: 1777-8.
3. Myles PS. Perioperative outcomes: are we asking the right questions? Can J Anesth 2016; 63: 138-41.

4. Boney $O$, Nathanson MH, Grocott MP, Metcalf L; Steering Group for the National Institute of Academic Anaesthesia/James Lind Alliance Anaesthesia and Peri-operative Care Setting Partnership. Differences between patients' and clinicians' research priorities from the Anaesthesia and Peri-operative Care Priority Setting Partnership. Anaesthesia 2017; 72: 1134-8.

5. McKeen DM, Banfield JC, McIsaac DI, et al. Top ten priorities for anesthesia and perioperative research: a report from the Canadian Anesthesia Research Priority Setting Partnership. Can J Anesth 2020; 67. Doi: https://doi.org/10.1007/s12630-02001607-6.

6. Top 10's of Priorities for Research. http://www.jla.nihr.ac.uk/top10-priorities/ (accessed February 2020).

7. Boney $O$, Bell $M$, Bell $N$, et al. Identifying research priorities in anaesthesia and perioperative care: final report of the Joint National Institute of Academic Anaesthesia/James Lind Alliance Research Priority Setting Partnership. BMJ Open 2015. DOI: https://doi.org/10.1136/bmjopen-2015-010006.

8. Elwyn $G$, Crowe $S$, Fenton $M$, et al. Identifying and prioritizing uncertainties: patient and clinician engagement in the identification of research questions. J Eval Clin Pract 2010; 16: 627-31.

9. Madden M, Morley R. Exploring the challenge of health research priority setting in partnership: reflections on the methodology used by the James Lind Alliance Pressure Ulcer Priority Setting Partnership. Res Involv Engagem 2016. DOI: https://doi.org/10. 1186/s40900-016-0026-y.

10. Nygaard A, Halvorsrud L, Linnerud S, Grov EK, Bergland A. The James Lind Alliance process approach: scoping review. BMJ Open 2019. DOI: https://doi.org/10.1136/bmjopen-2018-027473.

11. Staley $K$, Crowe $S$. More than a Top 10: How James Lind Alliance Priority Setting Partnerships transform research, people and organisations. NIHR Oxford Biomedical Research Centre, UK - September 2019. Available from URL: http://www. twocanassociates.co.uk/wp-content/uploads/2019/09/More-Thana-Top-10-Sep-19.pdf (accessed February 2020).

12. Crowe $S$, Giles $C$. Making patient relevant clinical research a reality. BMJ 2016 . DOI: https://doi.org/10.1136/bmj.i6627.

13. Gibson $F$. How far do research priority setting exercises influence what research is undertaken: a little, a lot, or not at all? Cancer Nurs 2019; 42: 89-90.

14. Chalmers I, Bracken MB, Djulbegovic B, et al. How to increase value and reduce waste when research priorities are set. Lancet 2014; 383: 156-65.

Publisher's Note Springer Nature remains neutral with regard to jurisdictional claims in published maps and institutional affiliations. 